\title{
Comparison of $1 \alpha-25$-dihydroxyvitamin D3 and IgE Levels between Allergic Rhinitis Patients and Healthy People
}

\author{
Mehmet Gokhan Demir ${ }^{1}$ \\ ${ }^{1}$ Department of ENT, Etımesgut State Hospıtal, Ankara, Turkey \\ Int Arch Otorhinolaryngol 2018;22:428-431.
}

\begin{abstract}
Address for correspondence Mehmet Gokhan Demir, Department of ENT, Etımesgut State Hospıtal, Ankara 06800, Turkey (e-mail: mgokhandemir@yahoo.com).
\end{abstract}

\begin{abstract}
Keywords

- allergic rhinitis

- immunoglobulin E

- vitamin D

- adult

Introduction Allergic rhinitis is one of the most common allergic diseases that cause a decrease in quality of life. Over the last decades, the increase in the number of cases is supposed to be a result of industrialization and lifestyle changes. Vitamin D, which is a steroid hormone, has a significant immunomodulatory and antioxidant role in the human body.

Objective The objective of the present study is to investigate the role of $1 \alpha-25$ dihydroxyvitamin D3 levels in allergic rhinitis patients and to compare them to the levels found in the healthy population.

Methods A total of 256 participants were included in the present study. Allergic rhinitis is diagnosed with the help of the Allergic Rhinitis and Its Impact on Asthma (ARIA) guidelines confirmed through skin prick tests. Control subjects were selected among people without allergy history, which was also confirmed through skin prick tests. The levels of $1 \alpha-25$-dihydroxyvitamin D3, immunoglobulin $\mathrm{E}(\mathrm{IgE})$, and eosinophil counts were measured in blood samples from both groups.

Results The mean value of serum levels of $1 \alpha$-25-hydroxyvitamin D3 was $25.5 \pm 3.74$ in the allergic rhinitis group, and $31.58 \pm 3.85$ in the control subjects. This difference reveals statistically-decreased levels in the allergic rhinitis group $(p<0.05)$. The total IgE levels are increased in the allergic rhinitis group $(p<0.05)$ and negatively correlated with the serum levels of $1 \alpha-25$-hydroxyvitamin $D 3(r=-0.259, p<0.05)$. Conclusion Lower serum levels of $1 \alpha-25$-hydroxyvitamin D3 were detected in the allergic rhinitis population. This data is also correlated to the $\lg E$ response in the study group. A supplement of $1 \alpha-25$-hydroxyvitamin D3 in deficient patients might be helpful to relieve symptoms and signs of allergic rhinitis, but further studies are needed.
\end{abstract}

\section{Introduction}

Allergic diseases such as asthma and allergic rhinitis are associated with an immune mediated reaction after exposure to the related allergen. Allergic rhinitis is a common health problem that interferes with the social and professional lives and decreases the quality of life of the patients. ${ }^{1}$ The prevalence of allergic rhinitis in Turkey is not known clearly, but in Europe the prevalence is $\sim 17$ and $25 \%$ of the population. ${ }^{2}$ Besides, prevalence can be changed according to the socioeconomic status of the population, and to the geographic and aeroaller-

received

May 31, 2017

accepted

February 18, 2018

published online

May 9, 2018

10.1055/s-0038-1649492. ISSN $1809-9777$. gen diversity in a specific region. Industrialized populations spend significant amounts of time indoors, which causes the deficiency of essential vitamins, such as vitamin D. Vitamin D is a steroid hormone primarily synthesized in the skin through a chemical reaction that is dependent on sun exposure. During its metabolism, the kidneys play an important role and convert it to its active form, known as $1 \alpha$-25-dihydroxyvitamin D3. This form of vitamin $D$ regulates both humoral and cellular immune responses in the human body. ${ }^{3,4}$ It is supposed that an increase in cases of allergic rhinitis is closely related to the deficiency of vitamin $\mathrm{D}$ in industrialized populations. There are several

Copyright @ 2018 by Thieme Revinter

Publicações Ltda, Rio de Janeiro, Brazil

License terms

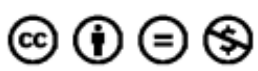


previous studies that have investigated this relationship. Wjst et $\mathrm{al}^{5}$ claimed that high levels of vitamin $\mathrm{D}$ cause allergic rhinitis, whereas Litonjua et $\mathrm{al}^{3}$ stated that low levels of vitamin D lead to allergic diseases. Besides, epidemiologic studies about vitamin D supplementation and allergic diseases have provided inconsistent results, causing confusion. ${ }^{6-8}$ Allergic rhinitis and its relationship with the active form of vitamin $D$, which has an important role in the metabolism have never been investigated in adult subjects, according to our knowledge. The objective of the present study is to investigate the levels of $1 \alpha$-25-dihydroxyvitamin D3 in allergic rhinitis patients and to compare them to the levels found in the normal population. We also want to investigate the relationship between the allergens and $1 \alpha-25$-dihydroxyvitamin D3 levels in the Ankara region in Turkey.

\section{Materials and Methods}

The present study was conducted at our hospital's otorhinolaryngology outpatient clinic (Etimesgut State Hospital ENT Department) between December 2014 and May 2015. A total of 256 patients was included in the study, and they were divided into two groups: patients with allergic rhinitis ( 65 male and 60 female) and control subjects (70 male and 61 female). The diagnosis of the allergic rhinitis patients was determined by the Allergic Rhinitis and Its Impact on Asthma (ARIA) 2008 guidelines. Allergic rhinitis patients were detected as presenting one or more of the following criteria: nasal obstruction, rhinorrhea, nasal itching and sneezing, all of which were supported by a skin prick test. ${ }^{9}$ The control group was composed of healthy volunteers who did not present any allergic symptoms and did not present any reaction to the skin prick test.

Subjects who presented chronic diseases such as diabetes, chronic renal insufficiency, vitamin D metabolism disorder, malignant diseases, asthma history, were overweight or obese (body mass index $[\mathrm{BMI}]>25$ ), used medications such as antihistamines or anti-allergy drugs, and were aged over 65 years old were excluded from the study. The BMI of all participants was calculated using the following formula: BMI = weight $(\mathrm{kg}) /[\text { height }(\mathrm{m})]^{2}$.

The allergy test included 15 different allergens supplied by Stallergenes Greer Laboratories (Antony, France). The allergen extracts were composed of pollen, weeds, grasses (maize, oat, wheat and barley), mold spores, mites (Dermatophagoides farina and Dermatophagoides pteronyssinus), epithelia, allergens derived from insects and other animals (feather mix, dog hair, cat hair and cockroach). Skin prick tests were applied to the forearms of all participants after cleaning the skin with alcohol. Each allergen was standardly diluted (1:50), and histamine hydrochloride $10 \mathrm{mg} / \mathrm{ml}$ and $50 \%$ glycerol-saline solutions were used as positive and negative controls respectively. The results were graded after 20 minutes as,,++++++ and ++++ , according to the level of erythema and the severity of the reaction.

A blood sample of each participant was collected at the end of the allergy test. Serum levels of the active form of vitamin-D ( $1 \alpha-25$-hydroxyvitamin D3) were measured using an enzyme immunoassay kit (Immunodiagnostic Systems
Holdings PLC, Tyne and Wear, UK). Afterwards, the levels detected in the allergic rhinitis patients and in the control subjects were compared.

Total serum levels of immunoglobulin E (IgE) and eosinophil counts from both groups were also measured using the IMMULITE 1000 Immunoassay System (Siemens, Munich, Germany). Peripheral blood eosinophil counts were conducted using fluorescent flow cytometry performed by a XT-1800i Automated Hematology Analyzer (Sysmex, Mundelein, IL, USA).

The data was compiled using the Statistical Package for the Social Sciences (SPSS, IBM Corp., Armonk, NY, US), version 22.0. The groups were compared through analysis of variance (ANOVA) and Pearson correlation analysis, and values of $p<0.05$ were considered statistically significant.

The present study was approved by our hospital's ethical committee (Dr. Lutfi Kurdar Kartal Education and Research Hospital Ethical Committee) and informed consent was taken from each participant.

\section{Results}

The study sample was composed of 256 subjects divided into two groups: a study group consisting of patients presenting allergic rhinitis ( 125 patients $=65$ male and 60 female $)$ and a control group (131 subjects $=70$ male and 61 female). The mean age of the study group was 44.5 years old, and the mean age of the control group was 42.4 years old. The groups were similar according to age, gender and BMI distribution $(p>0.05)$ (-Table 1). The mean value of serum $1 \alpha$-25-hydroxyvitamin D3 in the allergic rhinitis group was $25.5 \pm 3.74$ (female $=24.4$ \pm 3.31 and male $=24.9 \pm 3.29$ ). On the other hand, the mean value of serum $1 \alpha$-25-hydroxyvitamin D3 was $31.58 \pm 3.85$ (females $=31.12 \pm 3.62$ and males $=31.9 \pm 3.49$ ) in the control subjects. This difference reveals statistically-decreased levels in the allergic rhinitis group $(p<0.05)$ ( - Table 1 ). Eosinophil cationic protein (ECP) levels in both groups were similar, whereas total IgE levels were statistically increased in the allergic rhinitis group $(p<0.05)$. Additionally, the IgE levels were negatively correlated with the levels of serum $1 \alpha-25$ hydroxyvitamin D3 $(r=-0.259, p<0.05)$. The frequencies of positive skin prick test results of the allergic rhinitis patients are presented on - Table 2. According to this data, the most significant allergens are $D$. pteronyssinus, D.farina, Alternaria alternata and Alnus betulaceae (29.6, 28.6, 28.3 and 27.6 respectively). Although we have detected allergens in the allergic rhinitis group, we could not detect any correlation between vitamin D levels and the severity of the allergic reaction.

\section{Discussion}

Allergic rhinitis is one of the most common causes of the allergic condition, provoking a decrease in the quality of life. ${ }^{10}$ Allergens commonly leading to this response are pollen, dust, mite, and others. ${ }^{11}$ In the present study, the most frequent allergens in our region are $D$. pteronyssinus, D. farina, A. alternata and $A$. betulaceae, which had never been researched before. After exposure to these specific allergens, symptoms such as sneezing, nasal congestion, nasal itching and 
Table 1 The descriptives of allergic rhinitis and control subjects

\begin{tabular}{|c|c|c|c|c|c|c|c|}
\hline Variable & \multicolumn{3}{|c|}{ Allergic rhinitis $(n=125)$} & \multicolumn{3}{|c|}{ Control $(n=131)$} & $p$ value \\
\hline Age (years) & \multicolumn{3}{|c|}{$44.5 \pm 12.6$} & \multicolumn{3}{|l|}{$42.4 \pm 14.6$} & \multirow[t]{2}{*}{0.512} \\
\hline $\begin{array}{l}\text { (mean } \pm \mathrm{SD}) \\
(\text { minimum-maximum) }\end{array}$ & \multicolumn{3}{|l|}{$(32-57)$} & \multicolumn{3}{|l|}{$(28-58)$} & \\
\hline Gender (male/female) & \multicolumn{3}{|l|}{$65 / 60$} & \multicolumn{3}{|l|}{$70 / 61$} & 0.698 \\
\hline \multirow{2}{*}{$\begin{array}{l}\text { Serum } 1 \alpha-25 \text {-hydroxyvitamin D3 } \\
\text { (ng/mL) }\end{array}$} & \multicolumn{3}{|l|}{$25.5 \pm 3.74$} & \multicolumn{3}{|l|}{$31.58 \pm 3.85$} & $0.048^{*}$ \\
\hline & $\begin{array}{l}\text { Male } \\
24.9 \pm 3.29\end{array}$ & $\begin{array}{l}\text { Female } \\
24.4 \pm 3.31\end{array}$ & $p>0.895$ & $\begin{array}{l}\text { Male } \\
31.9 \pm 3.49\end{array}$ & $\begin{array}{l}\text { Female } \\
31.12 \pm 3.62\end{array}$ & $p>0.952$ & \\
\hline $\mathrm{ECP}(\mu \mathrm{g} / \mathrm{L})$ & \multicolumn{3}{|l|}{$25.2 \pm 31.5$} & \multicolumn{3}{|l|}{$24.9 \pm 29.28$} & 0.854 \\
\hline $\operatorname{lgE}(\mathrm{IU} / \mathrm{mL})$ & \multicolumn{3}{|l|}{$453.6 \pm 15.3$} & \multicolumn{3}{|l|}{$123.6 \pm 23.5$} & $0.01^{*}$ \\
\hline Body mass index $\left(\mathrm{kg} / \mathrm{m}^{2}\right)$ & \multicolumn{3}{|l|}{$21.5 \pm 3.45$} & \multicolumn{3}{|l|}{$22.1 \pm 3.05$} & 0.654 \\
\hline
\end{tabular}

Abbreviations: ECP, eosinophil cationic protein; IgE, immunoglobulin E; SD, standard deviation.

rhinorrhea, in any combination, could be seen in the clinical setting. ${ }^{12}$ According to the ARIA classification, allergic rhinitis is classified either as seasonal or perennial. The allergens affect the disease process, affecting the severity of the symptoms, which are classified as mild, moderate and severe, based on the disturbance in daily and social life. ${ }^{9}$ Patients with seasonal or perennial allergic rhinitis are less comfortable during allergen exposure in the Spring. The coincidence of this condition also fits with the reduced exposure to sunlight after winter, as in the Northern hemisphere. Turkey is located on a geographically fortunate area that provides enough exposure to sunlight during the summer, which is crucial for the biosynthesis of vitamin D. In order to prevent this effect, we have conducted the study between December and May, so we have measured the lowest vitamin D levels in both study groups.

According to our knowledge, the level of vitamin $D$ in allergic rhinitis populations, including the adult population in Turkey, had never been investigated. In the present study, we found

Table 2 The distribution of positive skin prick test in allergic rhinitis

\begin{tabular}{|l|l|}
\hline Specific allergen & Frequency (\%) \\
\hline Dermatophagoides pteronyssinus & 29.6 \\
\hline Dermatophagoides farina & 28.6 \\
\hline Alternaria alternata & 28.3 \\
\hline Alnus betulaceae & 27.6 \\
\hline Mixture of Cereals & 25.4 \\
\hline Grasses & 21.2 \\
\hline Feather mix & 20.9 \\
\hline Aspergillus mix & 20.1 \\
\hline Cladosporium & 18.2 \\
\hline Cat hair & 17.6 \\
\hline Dog hair & 17.5 \\
\hline Cockroach & 15.3 \\
\hline Penicillium mix & 14.2 \\
\hline Compositae & 10.7 \\
\hline A. fagacees & 8.3 \\
\hline
\end{tabular}

lower $1 \alpha$-25-dihydroxyvitamin D3 levels in the allergic rhinitis patients than in the healthy control subjects. There is only one study that has investigated the role of $1 \alpha$-25-dihydroxyvitamin D3 among children, and it revealed an increased risk of disease when there is vitamin D deficiency. ${ }^{13}$ There are also several studies that have researched the role of $1 \alpha-25$-dihydroxyvita$\min \mathrm{D} 3$ in the allergic response. Some of these studies supported the conclusion that a deficiency of this form of vitamin D leads to an increase in the allergic response, whereas other reports support the opposite. ${ }^{14-16}$ Therefore, the role of $1 \alpha$-25-dihydroxyvitamin D3 deficiency in allergic diseases is not currently clear. We believe that this situation is related to the assessment of the inactivated type of the vitamin $D$ form. These studies were also performed in the children population. Epidemiologic studies have shown that reference Vitamin D levels change with age. ${ }^{17}$ All of this data may with the result of inconsistent findings about the role of vitamin $D$ in allergic rhinitis.

Another problem in identifying the ideal level of vitamin D is the weight and fat storage of the participant. Vitamin $D$ is a fat soluble vitamin, and it can be present in lower levels in obese patients. ${ }^{18}$ In previous reports, there is not sufficient data in the methodology sections about the BMI values of the participants. This bias may be one of the reasons for the inconsistent results. To avoid this effect, we have excluded overweight and obese patients from the present study.

Vitamin D is a key hormone that modulates the immune response at the tissue level, participating both in innate and humoral immunity. Macrophages, dendritic cells (DCs) and lymphocytes possess receptors activated by the active form of vitamin $D\left(1 \alpha\right.$-25-dihydroxyvitamin D3). ${ }^{19} 1 \alpha$-25-dihydroxyvitamin $\mathrm{D} 3$ protects the activation and maturation of the DCs, resulting in the suppression of antigen presentation. The $1 \alpha-25-$ dihydroxyvitamin D3 level is important in the pathogenesis of the allergic diseases. Our findings support this data.

Environmental antigens, also known as allergens, cause increased synthesis of IgE. Immunoglobilin plays an important role in type-1 hypersensitivity reactions, such as allergic rhinitis, in which the IgE levels increased. The relationship between IgE levels and $1 \alpha$-25-dihydroxyvitamin D3 has been investigated before, and the results both in total and specific IgE levels. ${ }^{20,21}$ These inconsistent results may be related to the age of the selected study group, the duration of exposure to 
sunlight, and the changes in allergen diversity. In the present study, we have found increased levels of IgE in the allergic rhinitis patients. We have also found a relationship in which the IgE levels are increased when the $1 \alpha$-25-dihydroxyvitamin D3 levels decrease, which also supports our findings.

Vitamin D, which is crucial for biosynthesis and immune reactions in the human body, can be synthesized through a chemical reaction that is dependent on sun exposure. The role of vitamin $\mathrm{D}$ in allergic diseases such as allergic rhinitis has been investigated in previous reports, which have reached a clear conclusion. We thought that this is related to the selected experiment group (age, BMI) and the measurement of inactive vitamin $D$ in the tissue level. We tried to exclude this effect in our study, and confirmed the relationship between $1 \alpha-25$ dihydroxyvitamin D3 and allergic rhinitis in the adult population, but there were limitations in the present study as well. Firstly, the vitamin deficiency should be measured and followed-up throughout the year. Secondly, we should provide a $1 \alpha$-25-dihydroxyvitamin D3 supplement to the patients with $1 \alpha$-25-dihydroxyvitamin D3 deficiency and follow the therapeutic response of the allergic rhinitis.

\section{Conclusion}

In the present study, we have shown the decreased level of $1 \alpha$-25-dihydroxyvitamin D3 in allergic rhinitis in the adult population with specific features. Furthermore, this decrease is closely related with IgE levels. According to our knowledge, there is no similar study in the literature. We believe that future studies including large patient groups should be performed. These studies should cover the one-year measurement of $1 \alpha$-25-dihydroxyvitamin D3 and of vitamin D replacement in allergic rhinitis patients with vitamin $\mathrm{D}$ deficiency and detect their response to it.

\section{Declarations Section}

Ethical approval and consent to participate: The study was performed according to the rules of the Ethics Committee of Kartal Education and Research, with the reference number 2014.145.236. Informed consent was taken from each participant.

Availability of data and materials: Please contact author for data request.

\section{Author contribution}

MGD participated in the whole process of the study design, data analysis, data collection, statistical analyses, writing and drafting the article.

\section{Funding}

There was no financial support for this study.

Conflicts of interest

The authors have no conflicts of interest to disclose.

Acknowledgments

Not applicable.

\section{References}

1 Meltzer EO. The prevalence an medical ana economic of allergic rhinitis in the United States. J Allergy Clin Immunol 1997;99(06): 805-828

2 Bauchau V, Durham SR. Epidemiological characterization of the intermittent and persistent types of allergic rhinitis. Allergy 2005;60(03):350-353

3 Litonjua AA, Weiss ST. Is vitamin D deficiency to blame for the asthma epidemic? J Allergy Clin Immunol 2007;120(05):1031-1035

4 Holick MF. The photobiology of vitamin D3 in man. In: Kumar R(ed) Vitamin D: basic and clinical aspects. Martinus Nijhoff Publising, Boston; 1984:197-216

5 Wjst M, Dold S. Genes, factor X, and allergens: what causes allergic diseases? Allergy 1999;54(07):757-759

6 Miyake Y, Sasaki S, Tanaka K, Hirota Y. Dairy food, calcium and vitamin $D$ intake in pregnancy, and wheeze and eczema in infants. Eur Respir J 2010;35(06):1228-1234

7 Erkkola M, Kaila M, Nwaru BI, et al. Maternal vitamin D intake during pregnancy is inversely associated with asthma and allergic rhinitis in 5-year-old children. Clin Exp Allergy 2009;39(06):875-882

8 Bäck O, Blomquist HK, Hernell O, Stenberg B. Does vitamin D intake during infancy promote the development of atopic allergy? Acta Derm Venereol 2009;89(01):28-32

9 Bousquet J, Khaltaev N, Cruz AA, et al; World Health Organization; GA(2)LEN; AllerGen. Allergic Rhinitis and its Impact on Asthma (ARIA) 2008 update (in collaboration with the World Health Organization, GA(2)LEN and AllerGen). Allergy 2008;63(Suppl 86):8-160

10 Thompson AK, Juniper E, Meltzer EO. Quality of life in patients with allergic rhinitis. Ann Allergy Asthma Immunol 2000;85(05): 338-347, quiz 347-348

11 Boulet LP, Turcotte H, Laprise C, et al. Comparative degree and type of sensitization to common indoor and outdoor allergens in subjects with allergic rhinitis and/or asthma. Clin Exp Allergy 1997;27(01):52-59

12 Druce HM. Allergic and nonallergic rhinitis. Middleton EM Jr, Reed CE, Ellis EF, Adkinson NF Jr, Yunginger JW, Busse WW, eds. Allergy: Principles and Practice. 5th ed. St. Louis, Mo: Mosby Year-Book; 1998:1005-1016

13 Dogru M, Suleyman A. Serum 25-hydroxyvitamin D3 levels in children with allergic or nonallergic rhinitis. Int J Pediatr Otorhinolaryngol 2016;80:39-42

14 Liu X, Wang G, Hong X, et al. Gene-vitamin D interactions on food sensitization: a prospective birth cohort study. Allergy 2011;66 (11):1442-1448

15 Sharief S, Jariwala S, Kumar J, Muntner P, Melamed ML. Vitamin D levels and food and environmental allergies in the United States: results from the National Health and Nutrition Examination Survey 2005-2006. J Allergy Clin Immunol 2011;127(05):1195-1202

16 Wjst M, Hyppönen E. Vitamin D serum levels and allergic rhinitis. Allergy 2007;62(09):1085-1086

17 Mansbach JM, Ginde AA, Camargo CA Jr. Serum 25-hydroxyvitamin D levels among US children aged 1 to 11 years: do children need more vitamin D? Pediatrics 2009;124(05):1404-1410

18 Vlaykov A, Vicheva D, Stoyanov V. The role of vitamin D in the pathogenesis of allergic rhinitis and atopy. Turk Arch Otolaryngol 2013;51:63-66

19 Baeke F, Takiishi T, Korf H, Gysemans C, Mathieu C. Vitamin D: modulator of the immune system. Curr Opin Pharmacol 2010;10 (04):482-496

20 Hyppönen E, Berry DJ, Wjst M, Power C. Serum 25-hydroxyvitamin D and IgE - a significant but nonlinear relationship. Allergy 2009;64(04):613-620

21 Rothers J, Wright AL, Stern DA, Halonen M, Camargo CA Jr. Cord blood 25-hydroxyvitamin $D$ levels are associated with aeroallergen sensitization in children from Tucson, Arizona. J Allergy Clin Immunol 2011;128(05):1093-9.e1, 5 